Cipango Cahiers d'études japonaises

$21 \mid 2014$

Nouveaux regards sur les arts de la scène japonais II

\title{
Folklore, études folkloriques et arts de la scène au Japon
}

Jean-Michel Butel

\section{(2) OpenEdition}

1 Journals

Édition électronique

URL : https://journals.openedition.org/cipango/2179

DOI : $10.4000 /$ cipango. 2179

ISSN : 2260-7706

Éditeur

INALCO

Édition imprimée

Date de publication : 31 décembre 2014

ISSN : 1164-5857

Référence électronique

Jean-Michel Butel, «Folklore, études folkloriques et arts de la scène au Japon », Cipango [En ligne], 21 |

2014, mis en ligne le 26 septembre 2016, consulté le 30 juin 2021. URL : http://

journals.openedition.org/cipango/2179; DOI : https://doi.org/10.4000/cipango.2179

\section{(c) (i) (9)}

Cipango est mis à disposition selon les termes de la Licence Creative Commons Attribution - Pas d'Utilisation Commerciale 4.0 International. 


\title{
Folklore, études folkloriques et arts de la scène au Japon
}

\author{
Jean-Michel ButeL \\ CEJ, Inalco
}

Le texte de Hashimoto Hiroyuki - traduit pour l'article suivant - évoque les rapports pouvant exister entre les recherches ethnologiques et les arts de la scène. Relativement technique, il examine l'impact des lois de protection du patrimoine sur l'évolution des spectacles folkloriques. Ce faisant, il propose à la recherche une petite révolution : ne plus être obnubilée par la question de l'authenticité historique d'arts «traditionnels », mais considérer les stratégies des acteurs dans leur re-création permanente des spectacles folkloriques ${ }^{1}$.

L'ethnologie, et avec elle les études folkloriques ${ }^{2}$, $s^{\prime}$ intéressent depuis leurs débuts aux fêtes et aux spectacles qui les rythment. Ceci n'est d'ailleurs pas sans

1. Le texte date de 1994, son appel a été largement entendu depuis.

2. Si ces deux termes sont homophones en japonais, « ethnologie 》(minzokugaku 民族 学) et 《études folkloriques 》 (minzokugaku 民俗学) désignent bien deux disciplines différentes ayant chacune un patrimoine littéraire, une histoire scientifique, des méthodes, des concepts et des objectifs distincts. J'ai choisi la traduction « études folkloriques » pour rendre le second (littéralement « étude des us et coutumes populaires »), car ceci me semble mieux amener à comprendre qu'il s'agit d'un domaine du savoir s'intéressant aux coutumes, au «folklore » du pays auquel appartient le chercheur, si l'on compare à d'autres termes concurrents (« ethnologie du Japon » d'Anne Bouchy, « ethnofolklore » de Pierre-François Souyri, etc.). 


\section{CIPANGO}

24 Cahiers d'études japonaises n ${ }^{\circ} 21$

constituer un certain paradoxe. On sait qu'ethnologie et études folkloriques se posent très rapidement comme une nouvelle façon de faire de l'histoire : histoire des peuples oubliés par l'histoire occidentale, d'une humanité plurielle pour l'ethnologie ; histoire de groupes sociaux négligés par l'histoire officielle et nationale pour les études folkloriques. Groupes sociaux négligés par l'histoire, sans doute! Pourtant, dans le contexte de la formation et du renforcement des États-nations de la fin du $\mathrm{XIX}^{\mathrm{e}}$ siècle et de la première moitié du $\mathrm{XX}^{\mathrm{e}}$ siècle, les études folkloriques ont très vite milité pour une science non de la marge, mais du plus commun, $\mathrm{du} \ll$ peuple », et même, pour le dire avec les folkloristes japonais, du « peuple ordinaire » (jōmin 常民) dans ses activités les plus quotidiennes (nichijō seikatsu 日常生活). L'idée est qu'il existe un socle de culture, un substrat culturel (kisō bunka 基層文化) japonais ayant subsisté à toutes les avanies de l'histoire, une couche culturelle fondamentale dont on perçoit qu'elle est depuis peu mise à mal - par la modernisation de la société disait-on avant 1945, par la samouraïsation des mœurs depuis Meiji, rajouta-t-on après la défaite - mais qu'il est possible d'appréhender en observant la vie ordinaire du peuple et ses pratiques quotidiennes. Or la fête ne relève pas plus du quotidien, mais se situe plutôt résolument du côté de l'exceptionnel (le hare ハレ des folkloristes japonais) pour les populations « traditionnelles », l'état de fête perpétuel étant justement une caractéristique - dangereuse - des nouveaux urbains ${ }^{3}$.

La contradiction logique (focalisation sur le peuple ordinaire dans ses affaires quotidiennes/recherche sur le temps de la fête), si elle a été discutée, ne semble pas avoir gêné les chercheurs de façon rédhibitoire. À cela deux raisons selon nous, l'une liée à l'objet d'étude lui-même, la seconde à l'organisation de la connaissance à l'ère Meiji.

La fête, et les manifestations de tous ordres qu'elle accueille ${ }^{4}$, possèdent de toute évidence une force d'attraction qui leur est propre : l'exotisme qui y est à son comble et la prouesse, exceptionnelle, permettent descriptions croustillantes et clichés sensationnels. Elle cristallise et dévoile d'autre part des solidarités, des réseaux, des filiations, sans doute à l'œuvre dans le quotidien mais bien moins

3. C'est en tout cas ce qu'affirme Yanagita Kunio, dont nous parlerons plus longuement ci-après.

4. Nous nous garderons pour l'instant de les qualifier d'arts ou de spectacles : les critères de maîtrise technique ou l'opposition acteurs/public sont ici anachroniques. 
visibles. Elle permet enfin - et cette raison n'a jamais été aussi valide que pour les recherches contemporaines - un travail d'enquête fructueux malgré un engagement sur le terrain court, et s'avère ainsi d'une excellente rentabilité.

Mais il faut également, pour saisir la motivation des recherches folkloriques relatives aux arts performatifs, évoquer le contexte de la formation des disciplines scientifiques et la définition du patrimoine national à la fin du $\mathrm{XIX}^{\mathrm{e}}$ siècle ${ }^{5}$. De la même façon que le Japon se sentit poussé à définir une littérature nationale, et pour cela à effectuer un tri dans les productions passées et à venir ${ }^{6}$, d'intenses débats eurent lieu pour parvenir à une définition de ce qu'étaient et devaient être les «arts japonais ». De nombreuses manifestations artistiques furent mises à l'écart ; distinguées d'arts nobles, « nationaux », pouvant devenir académiques et professionnalisables ${ }^{7}$. Les folkloristes se donnèrent la mission, là encore, de recueillir les oubliés de l'histoire et de s'intéresser à ces représentations repoussées, de décrire ces performances, de militer pour que soient intégrés au patrimoine national les très nombreux spectacles qui, rejetés à Meiji, ne purent plus relever que du « folklore ». Ainsi naquit le registre des « spectacles du terroir » (kyōdo geinō 郷土芸能), remplacé après-guerre par celui, considéré comme plus « scientifique », des 《spectacles folkloriques» (minzoku geinō 民俗芸能). Celui-ci se construisit en relation étroite avec une sous-discipline des études folkloriques : les études folkloriques du spectacle. On décompterait aujourd'hui au Japon entre 20000 et 30000 manifestations par an, toutes catégories confondues. Si certaines ont une origine ancienne, remontant principalement au Moyen-Âge, la plupart sont reconnues être des créations de l'époque d'Edo ou de l'ère Meiji ${ }^{8}$.

5. Voir à ce propos le numéro 52 de la revue Ebisu - études japonaises, consultable en ligne : http://ebisu.revues.org/1569.

6. Emmanuel LOzERAND, Littérature et génie national : naissance d'une histoire littéraire dans le Japon du XIX $X^{e}$ siècle, Paris, les Belles Lettres, 2005.

7. Voir à ce sujet l'article de Suzuki Seiko, « Le gagaku, musique de l'Empire : Tanabe Hisao et le patrimoine musical comme identité nationale », Cipango, n ${ }^{\circ} 20,2013$, p. 95-139.

8. Pour un panorama des recherches, de leurs évolutions et de leurs problématiques, on pourra se référer à la collection Köza Nihon no minzokugaku 講座 日本の民俗学 (Série Recherches folkloriques japonaises), en particulier le volume 8 : Geijutsu to goraku no minzoku 芸術と娛楽の民俗 (Arts et plaisirs, 1999), et le volume 10 : Minzoku kenkyū no kadai 民俗研究の課題 (Les débats des études folkloriques, 2000), largement utilisés pour établir cette notice. 
26 Cahiers d'études japonaises $n^{\circ} 21$

\section{Les premières recherches}

Le premier travail dédié aux arts du spectacle locaux est réputé être un ouvrage de Yanagita Kunio publié en 1911, soit deux ans à peine après les premiers émois $\mathrm{du}$ « père des études folkloriques japonaises » devant la diversité culturelle qu'il constatait sur l'archipel ${ }^{9}$. Mais Yanagita Kunio est à cette époque un défricheur. Ses intuitions n’ont pas effet immédiat. Des études plus systématiques se développent la décennie suivante, et sont le fait de l'intérêt conjoint de folkloristes et d'historiens du théâtre académique. L'influence de la littérature sur son intérêt pour un théâtre, y compris populaire, n'est sans doute pas négligeable ${ }^{10}$.

La première Convention de danses et chants locaux ${ }^{11}$ est organisée par Yanagita Kunio et Kodera Yūkichi 小寺融吉 (1895-1945) en octobre 1925, à l'occasion de l'inauguration du bâtiment de la Japan Youth Association ${ }^{12}$. C'est la première fois que les arts folkloriques sont extirpés de leur contexte social pour être représentés sur une scène, dans le but de démontrer la richesse du Japon dans le domaine des spectacles folkloriques. Le mode de la convention apparaît comme une excellente occasion scientifique de réunir différentes manifestations en un même lieu, sous le regard des universitaires. C'est aussi un argument de poids pour convaincre le grand public - qui peut suivre les spectacles à la radio (NHK) de l'existence de véritables «spectacles » folkloriques. Le principe est poursuivi annuellement jusqu'en 1936. On verra dans le texte traduit qu'une Convention nationale des spectacles du terroir sera ressuscitée en 1950, toujours fidèle au terroir mais adoptant le terme générique de geinō pour désigner les spectacles ${ }^{13}$. Devenue Convention nationale des spectacles folkloriques ${ }^{14}$ en 1958, elle s'est tenue chaque année au même endroit jusqu'à aujourd'hui.

9. YANAGita Kunio, « Odori no ima to mukashi » 踊りの今と昔 (Danses, aujourd'hui et hier), Jinruigaku zasshi 人類学雑誌, vol. 27, Tōkyō, Jinrui gakkai 人類学会, 1911.

10. On pense pour l'époque à Shimazaki Tōson 島崎藤村 (1935) qui évoque des spectacles villageois, ou TANIZAKI Jun.ichirō 谷崎潤一郎 (1929) entre autres.

11. Kyōdo buyō to min.yō no kai 郷土舞踊と民謡の会.

12. Nihon seinen-kan 日本青年館.

13. Zenkoku kyōdo geinō taikai 全国郷土芸能大会.

14. Zenkoku minzoku geinō taikai 全国民俗芸能大会. 
La décennie allant de la seconde moitié des années 1920 au milieu des années 1930, celle d'un impérialisme de plus en plus conquérant est aussi, et ce n'est pas un hasard, une période d'intense organisation des réseaux scientifiques et intellectuels. L'Association des Arts populaires ${ }^{15}$ est créée en juillet 1927, suite à un voyage d'étude à Chichibu (Saitama) fin 1926 de plusieurs chercheurs rattachés à l'université de Waseda. L'équipe est dirigée par Kodera Yūkichi 小寺融吉, historien des arts de la scène japonais ayant étudié le théâtre occidental et auteur par la suite d'un travail conséquent sur les danses (buyō 舞踊) et chants (min.yō 民謡) populaires. L'Association compte les folkloristes les plus représentatifs et les plus actifs de l'époque : Yanagita Kunio, avec qui Kodera Yūkichi 小寺融吉 avait donc mis sur pied la première Convention, mais aussi Orikuchi Shinobu 折 口信夫, Hayakawa Kōtarō 早川孝太郎, Kindaichi Kyōsuke 金田一京助, Nakayama Tarō 中山太郎 et Nishitsunoi Masayoshi 西角井正慶. La présence de ces personnalités pourtant diverses, et parfois peu amies, dit assez la place centrale que prennent les arts de la scène dans la discipline folklorique. Le premier numéro d'Arts folkloriques ${ }^{16}$, organe de l'association, paraît sans tarder en janvier 1928. Il lance le modèle de l'article type dans le domaine : la présentation d'un spectacle donné en un lieu précis et de la documentation s'y rapportant. Si la publication prend fin en septembre 1932, son influence aura été grande. La revue Voyages et légendes (Tabi to densetsu 旅と伝説), fondée elle aussi en 1928, accueillera pour sa part des articles sur les arts performatifs folkloriques jusqu'en $1944^{17}$. Les recherches s'organisent alors autour de deux centres : l'université de Waseda, où se trouve Kodera Yūkichi 小寺融吉, qui penche pour une approche

15. Minzoku geijutsu no kai 民俗芸術の会, dont le nom révèle un certain flottement (geijutsu/geinō) dans le choix des concepts.

16. Minzoku geijutsu 民俗芸術.

17. Dans le monde des études folkloriques comme dans les autres disciplines, les revues foisonnent au Japon et n'ont pas toujours une durée de vie très longue. Les dernières années de la guerre interrompront d'ailleurs un grand nombre de titres. Certaines tentatives éphémères révèlent les dissensions : Orikuchi Shinobu 折口信夫, dont on sait la rivalité avec Yanagita Kunio, fondera la Société d'Études des Spectacles Folkloriques (Geinō gakkai 芸能学会), dont le journal (Geinō 芸能) ne paraîtra que de 1943 à 1944, lui aussi arrêté par la guerre. 
28 Cahiers d'études japonaises $n^{\circ} 21$

plus esthétique des arts performatifs ${ }^{18}$; l'université Kokugakuin et le Centre de recherche sur la culture japonaise animé par Orikuchi Shinobu 折口信夫, à qui Yanagita avait cédé plus ou moins explicitement le domaine ${ }^{19}$.

Mais l'autorité incontestée en la matière saura allier les deux perspectives (esthétique et folklorique) : diplômé de Waseda, influencé par Kodera Yūkichi 小 寺融吉 (1929) et Hayakawa Kōtarō 早川孝太郎 (1930), Honda Yasuji 本田 安次 n’a de cesse de chercher à établir la validité historique des spectacles folkloriques et d'attester de leur vitalité dans le monde contemporain. Son affectation dans un collège du département de Miyagi lui donne l'occasion de sillonner la région pour en étudier les différentes manifestations. Plusieurs publications en suivent, dont Yamabushi kagura, bangaku 山伏神楽 番楽 (Danses kagura et bangaku des anachorètes des montagnes, 1942), considéré comme un monument dans une œuvre au demeurant très large et très riche ${ }^{20}$.

\section{L'après-guerre}

La guerre avait arrêté la recherche et suspendu la représentation de spectacles folkloriques. La défaite fit ressentir l'urgence de travailler à la conservation de la culture japonaise. Les spectacles populaires vont bénéficier d'un arsenal juridique chargé de leur fournir les meilleures conditions pour qu'ils soient conservés au titre de patrimoine national. C'est de ces lois dont parlent Hashimoto Hiroyuki et

18. Voir KoderA Yūkichi 小寺融吉, Buyō no bigakuteki kenkyū 舞踊の美学的研 究 (Recherches esthétiques sur la danse japonaise), Tōkyō, Shun.yōdō 春陽堂, 1928 ; Geijutsu toshite no kagura no kenkyū 芸術としての神楽の研究 (Recherche sur le kagura comme art), Tōkyō, Chiheisha shobō 地平社書房, 1929.

19. L'article marquant l'intérêt d'ORIKUCHI Shinobu 折口信夫 pour ce thème s'intitule «Okina no hassei » 翁の発生 (La naissance d'Okina, 1928), et étudie les origines du personnage très présent au Japon d'Okina. Il sera publié dans le premier numéro de Minzoku geijutsu 民俗芸術.

20. Pour mieux cerner le personnage, voir par exemple ITō Jun 伊藤純, « Honda Yasuji no minzoku geinō-kan to sono kadai » 本田安次の民俗芸能観とその課題 (La vision des arts folkloriques de Honda Yasuji et les problèmes qu'elle pose), Minzoku geinō kenkyū 民俗芸能研究, $n^{\circ} 51,2011$, p. 1-23. 
nombre d'études contemporaines ${ }^{21}$. Après cinquante ans dominés par le souci de constituer un patrimoine et de le protéger, une nouvelle génération de chercheurs appelle en effet à un changement de paradigme : la quête d'un patrimoine national aussi authentique que possible, c'est-à-dire attesté par une longue histoire et labélisé par une agence gouvernementale, n'a-t-elle pas eu des effets décisifs sur l'évolution des spectacles eux-mêmes ? Ne serait-il pas temps de prendre en compte le dynamisme des arts performatifs dans le Japon contemporain, les contraintes et les choix qui président aux multiples inventions du siècle ? Hashimoto Hiroyuki propose pour cela de déplacer la focale des formes artistiques aux acteurs, dont on aurait trop oublié qu'ils sont les détenteurs de l'authenticité des arts de la scène.

\section{Bibliographie}

HAYAKAWA Kōtarō 早川孝太郎, Hanamatsuri 花祭, Tōkyō, Oka shoin 岡書 院, 1930.

HondA Yasuji 本田安次, Yamabushi kagura, bangaku 山伏神楽 番楽 (Danses kagura et bangaku des anachorètes des montagnes), Tōkyō, Asuka shobō, 1942.

Iтō Jun 伊藤純, «Honda Yasuji no minzoku geinō-kan to sono kadai » 本 田安次の民俗芸能観とその課題 (La vision des arts folkloriques de Honda Yasuji et les problèmes qu'elle pose), Minzoku geinō kenkyū 民俗芸能 研究, $\mathrm{n}^{\circ} 51,2011$, p. 1-23.

21. Pour un état des lieux récent et critique de la préservation du patrimoine au Japon et de l'effet des politiques internationales, voir l'article de KIKUCHI Akira, « La glocalisation de la protection du patrimoine folklorique : l'exemple des coutumes liées à la riziculture dans le nord de la péninsule de Noto », Ebisu, no 52, 2015, p. 233-289, trad. Jean-Michel Butel, consultable en ligne : http://ebisu.revues.org/1694. 
30 Cahiers d'études japonaises $n^{\circ} 21$

KIKUCHI Akira, « La glocalisation de la protection du patrimoine folklorique : l'exemple des coutumes liées à la riziculture dans le nord de la péninsule de Noto », Ebisu, no 52, 2015, p. 233-289, trad. Jean-Michel Butel, http:// ebisu.revues.org/1694.

KODERA Yūkichi 小寺融吉, Buyō no bigakuteki kenkyū 舞踊の美学的研究 (Recherches esthétiques sur la danse japonaise), Tōkyō, Shun.yōdō 春陽堂, 1928.

KOdera Yūkichi 小寺融吉, Geijutsu toshite no kagura no kenkyū 芸術としての 神楽の研究 (Recherche sur le kagura comme art), Tōkyō, Chiheisha shobō 地平社書房, 1929.

Komatsu Kazuhiko 小松和彦, Nomoto Kan.ichi 野本寬一 (dir.), «Geijutsu to goraku no minzoku »芸術と娛楽の民俗 (Arts et plaisirs), Köza Nihon no minzokugaku 講座 日本の民俗学 (Série Recherches folkloriques japonaises), vol. 8, Tōkyō, Yūzankaku 雄山閣, 1999.

Komatsu Kazuhiko 小松和彦, Nomoto Kan.ichi 野本寬一 (dir.), « Minzoku kenkyū no kadai »民俗研究の課題 (Les débats des études folkloriques), Kōza Nihon no minzokugaku 講座 日本の民俗学 (Série Recherches folkloriques japonaises), vol. 10, Tōkyō, Yūzankaku 雄山閣, 2000.

LOZERAND Emmanuel, Littérature et génie national : naissance d'une histoire littéraire dans le Japon du XIX siècle, Paris, les Belles Lettres, 2005.

Marquet Christophe, Nanta Arnaud, Nespoulous Laurent (dir.), Ebisu études japonaises : patrimonialisation et identités en Asie orientale, nº 52, 2015, http://ebisu.revues.org/1569.

ORIKUCHI Shinobu 折口信夫, 《Okina no hassei » 翁の発生 (La naissance d'Okina), Minzoku geijutsu 民俗芸術, vol. 1, Tōkyō, Chiheisha shobō 地平 社書房, 1928. 
Shimazaki Tōson 島崎藤村, Yoake mae 夜明け前 (Avant l'aube), Tōkyō, Shinchōsha 新潮社, 1935.

Suzuki Seiko, « Le gagaku, musique de l'Empire : Tanabe Hisao et le patrimoine musical comme identité nationale », Cipango, n 20, 2013, p. 95-139.

TANizaki Jun.ichirō 谷崎潤一郎, Tade kuu mushi 衤喰う虫, Tōkyō, Kaizōsha, 1929.

YANAGITA Kunio, 《Odori no ima to mukashi » 踊りの今と昔 (Danses, aujourd'hui et hier), Jinruigaku zasshi 人類学雑誌, vol. 27, Tōkyō, Jinrui gakkai 人類学会, 1911. 